\title{
Associations of centrally acting ACE inhibitors with cognitive decline and survival in Alzheimer's disease
}

\author{
Karim Fazal, ${ }^{*}$ Gayan Perera, ${ }^{*}$ Mizanur Khondoker, Robert Howard and Robert Stewart
}

\section{Background}

Cognitive improvement has been reported in patients receiving centrally acting angiotensin-converting enzyme inhibitors (C-ACEIS).

\section{Aims}

To compare cognitive decline and survival after diagnosis of Alzheimer's disease between people receiving C-ACEIS, non-centrally acting angiotensin-converting enzyme inhibitors (NC-ACEIS), and neither.

\section{Method}

Routine Mini-Mental State Examination (MMSE) scores were extracted in 5260 patients receiving acetylcholinesterase inhibitors and analysed against C-/NC-ACEl exposure at the time of Alzheimer's disease diagnosis.

\section{Results}

In the 9 months after Alzheimer's disease diagnosis, MMSE scores significantly increased by 0.72 and 0.19 points per year in patients on C-ACEIs and neither respectively, but deteriorated by 0.61 points per year in those on NC-ACEIS. There were no significant group differences in score trajectories from 9 to 36 months and no differences in survival.

\section{Conclusions}

In people with Alzheimer's disease receiving acetylcholinesterase inhibitors, those also taking C-ACEls had stronger initial improvement in cognitive function, but there was no evidence of longer-lasting influence on dementia progression.

\section{Declaration of interest}

R.S. has received research funding from Pfizer, Lundbeck, Roche, Janssen and GlaxoSmithKline.

\section{Copyright and usage}

(c) The Royal College of Psychiatrists 2017. This is an open access article distributed under the terms of the Creative Commons Non-Commercial, No Derivatives (CC BY-NC-ND) license.
As hypertension is associated with both an increased incidence of dementia, ${ }^{1}$ and conversion from mild cognitive impairment to dementia, ${ }^{2}$ attention has focused on treatments for hypertension as modifiers of cognitive decline. The influence of a number of antihypertensives has been investigated from mild cognitive impairment ${ }^{2,3}$ through to established dementia, ${ }^{4}$ with some agents associated with a lowered risk, but negative or inconsistent results from clinical trials. ${ }^{5-9}$ Angiotensin-converting enzyme inhibitors (ACEIs) have attracted interest in Alzheimer's disease specifically; however, not all ACEIs cross the blood-brain barrier which may give rise to heterogeneity in effects. In one study of community residents, reduced cognitive decline was found in those receiving centrally active inhibitors (centrally acting angiotensin-converting enzyme inhibitors (C-ACEIs)) compared with non-centrally active inhibitors (non-centrally acting angiotensin-converting enzyme inhibitors (NC-ACEIs)) and calcium channel blockers. ${ }^{10}$ Studies of dementia incidence have been mixed, ${ }^{11-15}$ but C-ACEI receipt was associated with slower cognitive decline in dementia over 6 months, ${ }^{4}$ and C-ACEIs have recently been recommended for slowing dementia progression in elderly patients with hypertension. ${ }^{16}$ Given the paucity of evidence in this field, we analysed a large, prospective clinical database of people receiving dementia assessment and acetylcholinesterase treatment, to compare cognitive function trajectories and survival among C-ACEI users with those using NC-ACEIs or neither agent.

\section{Method}

\section{Study setting and data source}

A retrospective observational study was conducted using data from the South London and Maudsley NHS Foundation Trust

*These authors contributed equally to this work.
(SLaM) Biomedical Research Centre (BRC) case register. SLaM is one of Europe's largest mental healthcare providers, serving a geographic catchment of over 1.2 million residents in four South London boroughs (Lambeth, Lewisham, Southwark and Croydon). In 2007-2008, the Clinical Record Interactive Search (CRIS) application was developed with National Institute for Health Research (NIHR) funding to provide researcher access to anonymised copies of SLaM's electronic health record within a robust governance framework. ${ }^{17}$ The SLaM BRC case register has been described in detail, ${ }^{18}$ and has supported a range of studies, ${ }^{19,20}$ including several longitudinal studies of large dementia cohorts. ${ }^{21-23}$ Data are currently archived in CRIS on over 270000 cases with a range of mental disorders and the database has full approval for secondary analysis (Oxford Research Ethics Committee C, reference 08/ H0606/71+5). Data from CRIS have been extensively supplemented through natural language processing applications using Generalised Architecture for Text Engineering (GATE) software, applying information extraction techniques to derive structured information from the extensive text fields held in the mental health record. $^{24}$

\section{Sample}

All cases with Alzheimer's disease (ICD-10 F00.x) diagnosed at any point between 1 January 2000 and 31 May 2014 were ascertained in CRIS using a combination of data from structured fields for primary and secondary diagnoses, and a specific natural language processing application extracting text associated with diagnostic statements in open text fields. ${ }^{24}$ The sample was restricted to patients who received a first diagnosis of Alzheimer's disease during the study period, and who were commenced on treatment with an acetylcholinesterase inhibitor. Those who received angiotensin receptor blockers at the time of Alzheimer's disease diagnosis were also excluded - this was partly 
to maximise homogeneity of the sample and partly because those receiving this treatment had the most routine data on cognitive decline.

\section{Measurements}

The index date for defining exposure and confounding variables was the date of the first recorded Alzheimer's disease diagnosis. Demographic information obtained included age at diagnosis, age at death, gender, ethnicity (European and nonEuropean) and index of multiple deprivation (2010 projections from the UK Census) for each patient's neighbourhood (UK lower super output area) at the time of diagnosis. Health of the Nation Outcome Scales (HoNOS) are routinely administered in UK mental health services and recorded as structured data on the electronic health record. HoNOS item scores and dates were extracted for the period of 6 months before and after the diagnosis date, and the closest scores in time were included in analyses.

Medications received were extracted from structured medication fields supplemented by natural language processing applications applied to open text fields. ${ }^{24}$ C-ACEIs were defined as perindopril, ramipril, trandolapril, captopril, fosinopril, lisinopril, prinivil, monopril; NC-ACEIs were defined as enalapril, imidapril, cilazapril, quinapril, moexipril. Initial and most recent dates were ascertained for when these medications were recorded and exposure was defined on the basis of recorded medication use at or before the Alzheimer's disease diagnosis. The analysis excluded patients who were recorded as receiving both C-ACEIs and NC-ACEIs (because these groups were too small to analyses separately), and a third group of patients were defined with no recorded ACEI use. Use of antipsychotic or antidepressant medication was also ascertained at the time of the Alzheimer's disease diagnosis. Use of other antihypertensive medication (vasodilators, diuretics, calcium channel blockers, beta-blockers, alpha-blockers) was also ascertained at the time of Alzheimer's disease diagnosis.

The primary outcome was change in cognitive function as estimated from recorded Mini-Mental State Examination (MMSE) scores. In British dementia treatment services, cognitive function continues to be primarily monitored in routine clinical practice using the MMSE, a 30-point measure of global function in wide use. ${ }^{25}$ MMSE numerator and denominator scores and dates of assessment for the cohort were obtained from structured fields and a bespoke natural language processing application. ${ }^{24}$ According to the availability of follow-up data and to minimise the influence of differential attrition, MMSE scores were restricted to those recorded within 3 years after the Alzheimer's disease diagnosis date, and the analysed sample was restricted to patients with more than one MMSE score recorded. Date of death was obtained from death registry information from the Office for National Statistics (ONS) linked to CRIS.

\section{Statistical analysis}

Initially, generalised additive models for location, scale and shape (GAMLSS) $^{26}$ were used to visualise the shape of MMSE score trajectories for the three cohorts - those who were receiving C-ACEIs, those who were receiving NC-ACEIs and those who were not receiving any ACEIs. An advantage of GAMLSS is that they are not restricted to a linearity assumption: important because of the potential association between cognitive decline and time since Alzheimer's disease diagnosis and acetylcholinesterase inhibitor initiation. GAMLSS are parametric, in that they require a parametric distribution assumption for the response variable, and 'semi' in the sense that the modelling of the parameters of the distribution, as functions of explanatory variables, may involve using non-parametric smoothing functions. Although GAMLSS output provided a helpful way of visualising the pattern of cognitive decline within the observation window, it did not permit analyses of predictive covariates, confounding and effect modification. By inspecting the curves derived from GAMLSS, it was concluded that it would be appropriate to use parametric methodology in the form of a two-piecewise linear mixed model to estimate cognitive change and its predictors, using the two time components: Segment 1 for the 0-9 months after diagnosis and Segment 2 for the 9-36 months after diagnosis. Slopes and slope differences were obtained using linear mixed estimation methodology. Two-piecewise model estimates were adjusted for age, gender, ethnicity, other antihypertensives, antipsychotic, antidepressant receipt and HoNOS subcomponents including agitated behaviour, self-injury, problem drinking or drug use, physical illness, hallucinations or delusions, depressed mood, relationship problems, daily living problems, living conditions problems and occupational problems.

Survival analyses were carried out for mortality as an outcome up to a census point of 8 July 2014. Kaplan-Meier survival curves were constructed using STATA 13 software, and a log-rank test was carried out to test associations with ACEI use.

\section{Results}

Fourteen patients were excluded who were receiving both C-ACEI and NC-ACEI agents and an additional ten patients who had only one recorded MMSE score since diagnosis. Furthermore, 27 patients receiving C-ACEIs, 2 patients receiving NC-ACEIs and 296 patients receiving neither were excluded as they received angiotensin receptor blockers at the time of Alzheimer's disease diagnosis. The analysed samples comprised 1207 patients receiving C-ACEIs, 143 receiving NC-ACEIs and 3910 receiving neither. Characteristics of the samples are summarised in Table 1. In summary, the group receiving NC-ACEIs were more likely to be to be male, to be of non-European ethnicity and to be rated as having hallucinations or delusions than those receiving C-ACEIs or neither. There were no significant individual group differences in age (ANOVA post hoc test). However, a post hoc test showed a significant difference in mean deprivation score between $\mathrm{C}$-ACEI group and the group receiving neither ACEI $(P=0.008)$ and between the NC-ACEI group and those receiving neither ACEI $(P=0.041)$. Antipsychotic and antidepressant uses were more common in patients receiving C-ACEIs. Both the C-ACEI and NC-ACEI groups were rated as having more severe physical health problems on the HoNOS than those receiving neither. Importantly, although there were differences in the timing and number of some measurement points, there was no difference in MMSE score closest to diagnosis between the three groups.

Figure 1 displays GAMLSS curve for trajectories of MMSE scores by ACEI receipt, and Tables 2 and 3 summarise the results of the linear mixed estimations from two-piecewise estimations model comapring slopes at 0-9 months and 9-36 months after Alzheimer's disease diagnosis. Analysis with adjustments showed the NC-ACEI group to have slightly higher MMSE scores at Alzheimer's disease diagnosis compared with the other two groups. In patients who received C-ACEIs, MMSE scores improved over the first 9 months, with a slope coefficient of 0.72 (points per year), whereas they declined in those receiving NC-ACEIs and slightly improved in those that received neither ACEI, with slope coefficients of -0.61 and 0.19 respectively. For patients receiving C-ACEIs, these slopes were significantly different both compared with those receiving NC-ACEIs (slope difference 1.33, $P=0.028$ ) 


\begin{tabular}{|c|c|c|c|c|}
\hline Variable & $\begin{array}{c}\text { C-ACEIS only } \\
(n=1207)\end{array}$ & $\begin{array}{l}\text { NC-ACEIS only } \\
(n=143)\end{array}$ & $\begin{array}{l}\text { Neither agent } \\
\quad(n=3910)\end{array}$ & $P^{a}$ \\
\hline Mean age at diagnosis (s.d.), years & $80.5(7.1)$ & $81.4(7.0)$ & $80.2(8.0)$ & 0.116 \\
\hline Age at diagnosis, $n(\%)$ & & & & 0.095 \\
\hline$<65$ & $27(2.2)$ & $1(0.7)$ & $170(4.3)$ & \\
\hline $65-69$ & $62(5.1)$ & $7(4.8)$ & $177(4.5)$ & \\
\hline $70-74$ & $130(10.8)$ & $15(11)$ & $420(10.7)$ & \\
\hline $75-79$ & $266(22.0)$ & $22(15.1)$ & 735 (18.8) & \\
\hline $80-84$ & $329(27.3)$ & $49(33.6)$ & $1021(26.1)$ & \\
\hline $85-89$ & $280(23.2)$ & $29(20.5)$ & $892(22.8)$ & \\
\hline 90 and over & $113(9.4)$ & $20(14.4)$ & $495(12.7)$ & \\
\hline Age at death, $n(\%)$ & & & & 0.127 \\
\hline$<65$ & $1(0.1)$ & $0(0)$ & $24(0.6)$ & \\
\hline $65-69$ & $7(0.6)$ & $0(0)$ & $26(0.7)$ & \\
\hline $70-74$ & $22(1.8)$ & $3(2.7)$ & $83(2.1)$ & \\
\hline $75-79$ & $73(6.0)$ & $7(4.8)$ & $201(5.1)$ & \\
\hline $80-84$ & $134(11.1)$ & $15(10.3)$ & $433(11.1)$ & \\
\hline $85-89$ & $151(12.5)$ & $23(16.4)$ & $583(14.9)$ & \\
\hline 90 and over & $145(12.0)$ & $22(15.1)$ & $594(15.2)$ & \\
\hline Alive at end of follow-up, $n$ (\%) & $674(55.8)$ & $73(50.7)$ & $1966(50.3)$ & 0.013 \\
\hline Ethnicity, $n$ (\%) & & & & $<0.001$ \\
\hline European & $961(79.6)$ & $109(76.0)$ & $3270(83.6)$ & \\
\hline Non-European & $246(20.4)$ & $34(24.0)$ & $640(16.4)$ & \\
\hline Gender, $n(\%)$ & & & & 0.009 \\
\hline Male & $442(36.6)$ & $55(38.4)$ & 1301 (33.3) & \\
\hline Female & $765(63.4)$ & $88(61.6)$ & $2609(66.7)$ & \\
\hline Mean (s.d.) index of multiple deprivation & $27.1(11.2)$ & $28.1(11.2)$ & $26.0(11.2)$ & $<0.001$ \\
\hline Mean ACE inhibitors follow-up years (s.d.) & $0.8(1.5)$ & $1.1(1.8)$ & & 0.024 \\
\hline \multicolumn{5}{|l|}{ Medications received, $n$ (\%) } \\
\hline Antihypertensives & $617(51.1)$ & $80(55.9)$ & $1684(43.1)$ & $<0.001$ \\
\hline Antipsychotics & $328(27.2)$ & 39 (27.3) & 995 (25.4) & 0.884 \\
\hline Antidepressants & $459(38.0)$ & $48(33.6)$ & $1261(32.3)$ & 0.695 \\
\hline \multicolumn{5}{|l|}{ MMSE scores, mean (s.d.) } \\
\hline Score closest to diagnosis & $19.0(6.3)$ & $19.9(6.9)$ & $19.1(6.5)$ & 0.71 \\
\hline Number of MMSE scores per patient & $4.6(3.8)$ & $4.7(4.1)$ & $4.0(3.6)$ & $<0.001$ \\
\hline $\begin{array}{l}\text { Interval between diagnosis and MMSE } \\
\text { score closest to diagnosis, years }{ }^{\mathrm{b}}\end{array}$ & $0.0(0.7)$ & $-0.1(0.7)$ & $-0.1(0.8)$ & 0.033 \\
\hline $\begin{array}{l}\text { Interval between first mention of an } \\
\text { ACE inhibitor and diagnosis, years }{ }^{c}\end{array}$ & $-0.1(1.7)$ & $-0.5(2.0)$ & & 0.041 \\
\hline \multicolumn{5}{|l|}{ Mean (s.d.) HoNOS subscale score } \\
\hline Agitated behaviour & $0.7(1.0)$ & $0.7(1.0)$ & $0.7(1.0)$ & 0.652 \\
\hline Self-injury & $0.1(0.4)$ & $0.1(0.3)$ & $0.1(0.3)$ & 0.481 \\
\hline Problem drinking/drug use & $0.1(0.4)$ & $0.1(0.3)$ & $0.1(0.4)$ & 0.248 \\
\hline Cognitive problems & $2.4(0.8)$ & $2.4(0.8)$ & $2.4(0.9)$ & 0.937 \\
\hline Physical illness & $1.6(1.1)$ & $1.6(1.2)$ & $1.4(1.2)$ & $<0.001$ \\
\hline Hallucinations/delusions & $0.4(0.9)$ & $0.5(0.9)$ & $0.4(0.8)$ & 0.367 \\
\hline Depressed mood & $0.6(0.8)$ & $0.6(0.8)$ & $0.6(0.8)$ & 0.289 \\
\hline Other mental problems & $0.8(1.0)$ & $0.8(1.0)$ & $0.8(1.0)$ & 0.287 \\
\hline Relationship problems & $0.6(0.9)$ & $0.7(0.9)$ & $0.6(0.9)$ & 0.281 \\
\hline Daily living problems & $1.8(1.1)$ & $1.8(1.1)$ & $1.7(1.2)$ & 0.843 \\
\hline Living conditions problems & $0.4(0.8)$ & $0.5(0.9)$ & $0.4(0.8)$ & 0.609 \\
\hline Occupational problems & $1.1(1.1)$ & $1.2(1.1)$ & $1.0(1.1)$ & 0.143 \\
\hline Mean (s.d.) interval between HoNOS and diagnosis, years & $0.1(0.6)$ & $0.2(0.7)$ & $0.2(0.8)$ & 0.082 \\
\hline \multicolumn{5}{|c|}{$\begin{array}{l}\text { C-ACEIS, centrally acting angiotensin converting enzyme inhibitors; NC-ACEIS, non-centrally acting angiotensin converting enzyme inhibitors; MMSE, Mini-Mental State Examination; } \\
\text { ACE, angiotensin converting enzyme; HoNOS, Health of the Nation Outcome Scales. } \\
\text { a. Difference in means between three groups tested using one-way ANOVA test, difference in means between two medication groups were tested using independent sample } t \text {-tests, } \\
\text { differences in frequencies tested using chi-squared tests. } \\
\text { b. Negative values indicating that MMSE scores recorded before Alzheimer's disease diagnosis. } \\
\text { c. Negative values indicating ACE inhibitor medication recorded before Alzheimer's disease diagnosis. }\end{array}$} \\
\hline
\end{tabular}

and compared with those who received neither ACEI (slope difference $0.53, P=0.037$ ). However, there were no significant group differences in slopes for the 9-36 month period after diagnosis. Figure 2 displays GAMLSS curve for trajectories of MMSE scores by other antihypertensive use for those who did not receive any ACEIs. There were no significant differences in slopes between those who received other antihypertensive medication and not receiving such medication. Likewise, as shown in Fig. 3, there was no observable group difference in survival. Log-rank tests for equality of survivor functions gave $P$-values of 0.82 and 0.84 , respectively, for comparisons between C-ACEIs and NC-ACEIs, and between C-ACEIs and no ACEI. 

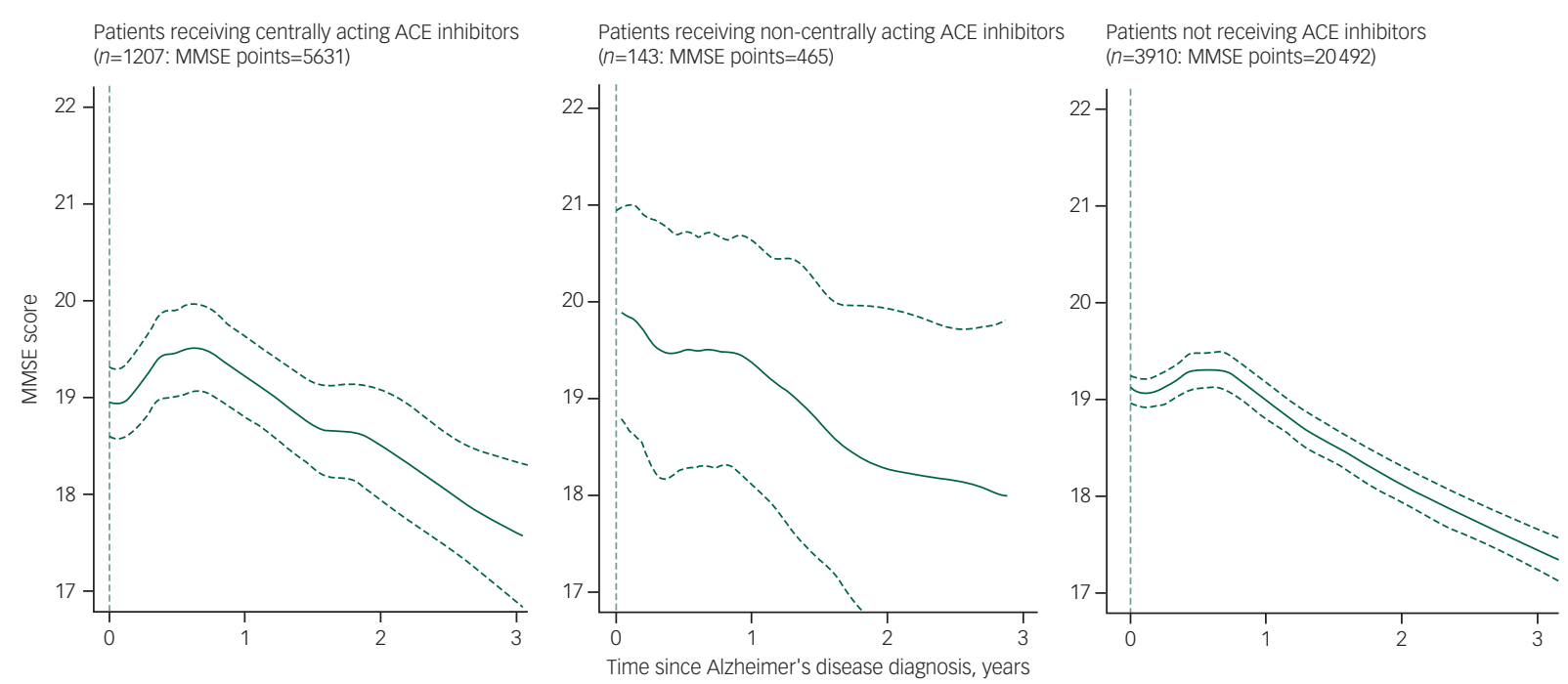

Fig. 1 Comparison of longitudinal change in Mini-Mental State Examination (MMSE) in the samples using generalised additive models for location scale and shape (GAMLSS) methodology. ACE, angiotensin converting enzyme.

\begin{tabular}{|c|c|c|c|c|}
\hline MMSE slope & Received C-ACEI & $\begin{array}{c}\text { Did not receive } \\
\text { ACEI }\end{array}$ & $\begin{array}{l}\text { MMSE slope difference } \\
\text { (MMSE per year) }\end{array}$ & $\begin{array}{l}P \text { for group } \\
\text { difference }\end{array}$ \\
\hline First MMSE slope 0-9 months & 0.72 (0.89 to 0.55$)$ & 0.19 (0.12 to 0.21$)$ & 0.53 (0.05 to 1.01) & 0.037 \\
\hline Second MMSE slope 9-36 months & $-0.81(-1.17$ to -0.45$)$ & $-0.68(-1.54$ to 0.18$)$ & $-0.13(-1.03$ to 0.77$)$ & 0.626 \\
\hline
\end{tabular}

\begin{tabular}{|c|c|c|c|c|}
\hline MMSE slope & Received C-ACEIS & Received NC-ACEIS & $\begin{array}{l}\text { MMSE slope difference } \\
\text { (MMSE per year) }\end{array}$ & $\begin{array}{l}P \text { for group } \\
\text { difference }\end{array}$ \\
\hline First MMSE slope 0-9 months & 0.72 (0.89 to 0.55$)$ & $-0.61(-0.21$ to -1.02$)$ & $1.33(0.25$ to 1.41$)$ & 0.028 \\
\hline Second MMSE slope 9-36 months & $-0.81(-1.17$ to -0.45$)$ & $-0.63(-1.36$ to 0.10$)$ & $-0.18(-1.50$ to -1.14$)$ & 0.531 \\
\hline
\end{tabular}

\section{Discussion}

In what we believe to be the largest investigation to date of antihypertensive agents and dementia progression, we compared trajectories of routinely measured cognitive function between patients receiving C-ACEIs and NC-ACEIs at the time of Alzheimer's disease diagnosis and those receiving neither type of medication. An initial improvement in cognitive function was found in patients receiving C-ACEIs over the first 9 months after diagnosis compared with deteriorations in the NC-ACEIs group. However, there was no evidence of longer-term slope differences and no differences in survival between the groups.

As previously summarised, several studies have suggested that C-ACEIs may influence cognitive function trajectories in people without dementia. The Cardiovascular Health Study Cognition Sub-Study, which followed up people with hypertension for 6 years, found no significant differences in cognitive decline between ACEIs and other antihypertensives, but did find significantly reduced cognitive decline in people receiving C-ACEIs: reduced by $65 \%$ per year of exposure in this group compared with those receiving NC-ACEIs. ${ }^{11}$ A Japanese study found no difference in Alzheimer's disease incidence associated with antihypertensive drugs as a whole, but did find a significantly lower risk in a subgroup analysis of captopril and perindopril (C-ACEIs) compared with enalapril and imidapril (NC-ACEIs). ${ }^{13}$ Also, in secondary analyses of the Perindopril Protection Against Recurrent Stroke Study (PROGRESS), there was a reduced risk of cognitive decline in the treatment group. ${ }^{27}$ There has been less investigation of associations between ACEIs and dementia progression, although one study found that C-ACEIs, compared with other antihypertensive drugs, were associated with a reduced rate of cognitive decline in hypertensive patients with mild to moderate Alzheimer's disease, although all participants had stable and comparable blood pressure levels, ${ }^{10}$ suggesting a mechanism other than lowered blood pressure. Our findings of initial improvement in cognition function in patients with C-ACEIs are also consistent with findings from two smaller research cohorts of reduced 12-month functional decline in patients with Alzheimer's disease, ${ }^{28,29}$ with reduced 6-month cognitive decline in patients with Alzheimer's, vascular and mixed dementia, ${ }^{21}$ and with reduced cognitive decline in patients with Alzheimer's disease with specific ACE haplotypes. ${ }^{9}$

The mechanisms by which C-ACEIs might influence dementia outcomes remain unclear. As previously described, ACEIs have been highlighted as potentially detrimental for cognitive function 

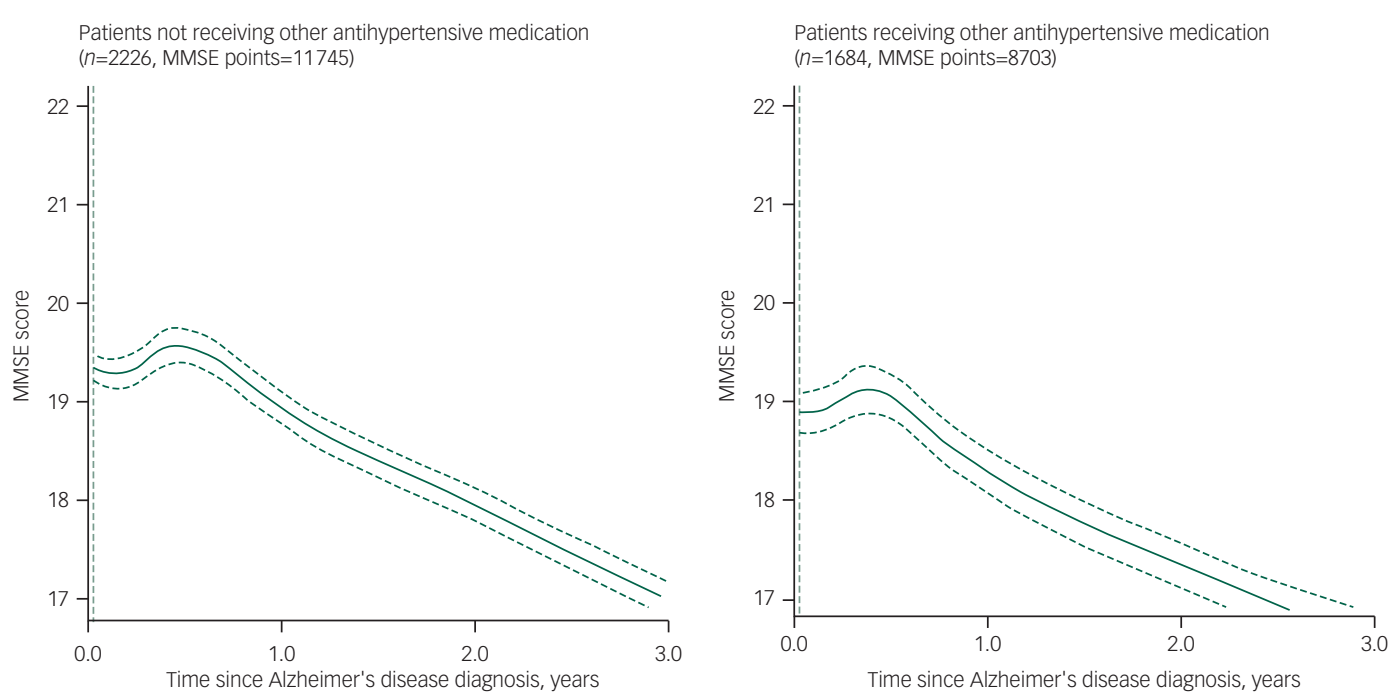

Fig. 2 Comparison of longitudinal change in Mini-Mental State Examination (MMSE) among patients not receiving angiotensin converting enzyme inhibitors stratified by other antihypertensive use.

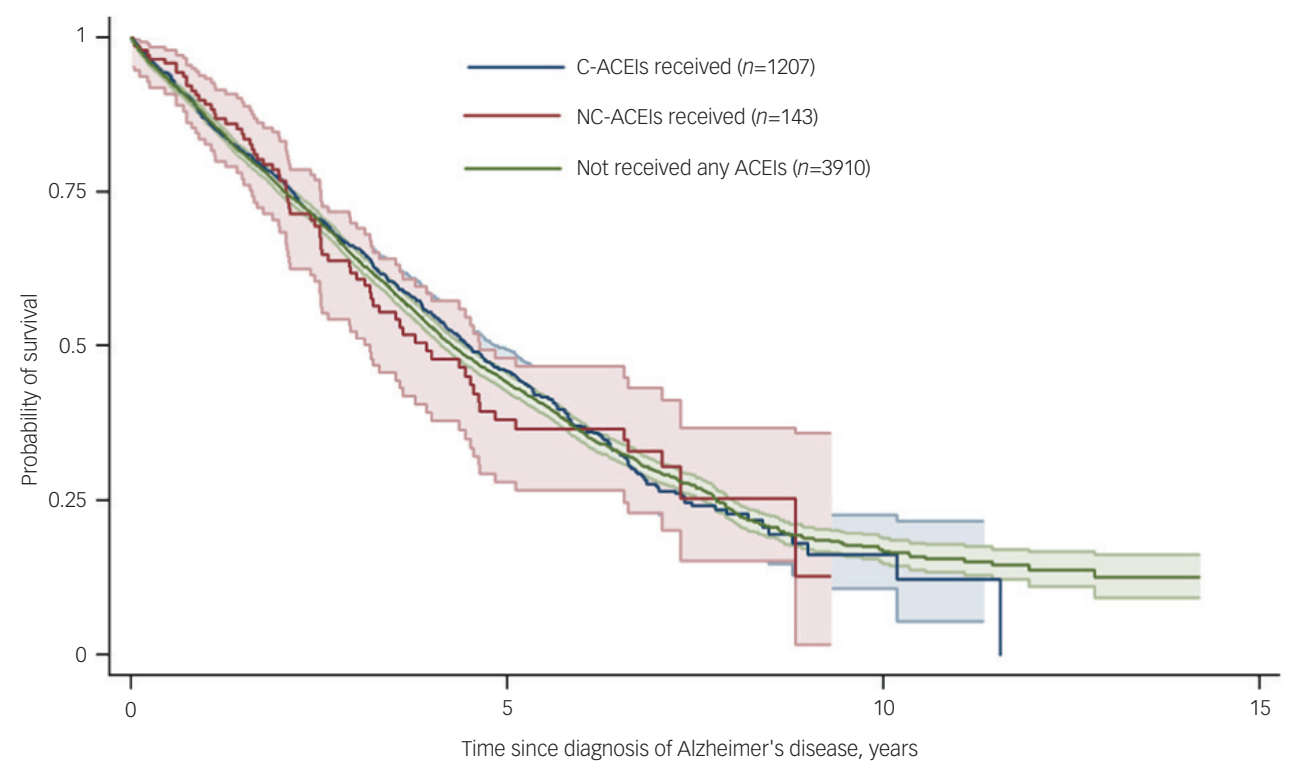

Fig. 3 Kaplan-Meier survival curves comparing exposure groups. ACEIS, angiotensin converting enzyme inhibitors; C-ACEls, centrally acting angiotensin converting enzyme inhibitors; NC-ACEIS, non-centrally acting angiotensin converting enzyme inhibitors.

because of ACE degrading amyloid and observations of an increase of A $\beta 1-42$ in mice associated with ACEI administration. ${ }^{30}$ However, there are also a number of potential reasons why these agents may be protective. First, reduced cerebral blood flow has been observed in Alzheimer's disease, ${ }^{31}$ and C-ACEIs may improve this by inhibiting the production of angiotensin II, a vasoconstrictor; this is supported by perivascular staining of both angiotensin II and ACE seen in patients with Alzheimer's disease. ${ }^{32}$ Second, the renin angiotensin system within the brain has an effect on neural plasticity and long-term potentiation, ${ }^{33}$ and it has been proposed that C-ACEIs through preventing activation of this system inhibits the release of inflammatory cytokines which may be involved in the neurodegenerative process. ${ }^{34,35}$ The renin angiotensin aldosterone system (RAAS) acts throughout the body, including the central nervous system, ${ }^{36}$ and there is growing evidence of its importance in neurodegenerative disorders including Alzheimer's disease and Parkinson's disease. ${ }^{37}$ Perindopril, but not other ACEIs, significantly inhibited hippocampal ACE, preventing cognitive impairment in mouse models of Alzheimer's disease, ${ }^{38,39}$ and a study in hypertensive rats found that lifelong treatment with captopril (C-ACEI) significantly reduced impairment in learning and memory compared with hydralazine, despite equal blood pressure control in both groups. ${ }^{40}$ Third, angiotensin II inhibits release of acetylcholine ${ }^{41}$ and C-ACEIs might increase acetylcholine activity (and thus improve cognitive function) by reducing angiotensin II levels. ${ }^{11}$ Yamada et al demonstrated an enhancing effect of perindopril (C-ACEI) on extracellular acetylcholine levels in the perirhinal cortex in rats with chronic cerebral hypoperfusion. ${ }^{42}$

The fact that we were only able to observe an improvement over a 9-month period in people with Alzheimer's disease and that the longer-term decline trajectory was unaffected, favours the third and more 'symptomatic' of these mechanisms. It should be borne in mind that we only ascertained ACEI use at the time of diagnosis and did not attempt to follow up its continuation. From the same data resource, we have previously reported an improvement in routinely measured cognitive function during the first 6-9 months following initiation of acetylcholinesterase inhibitor medication in dementia. ${ }^{21}$ All patients included in the 
study reported here were receiving acetylcholinesterase inhibitor treatment, so that the more marked improvement in MMSE score trajectories in those also receiving C-ACEIs compared with patients who did not receive ACEIs cannot be attributed to cholinesterase inhibition alone. Furthermore, the relative improvement in patients receiving C-ACEIs compared with those receiving NC-ACEIs renders a generic effect of hypertension or antihypertensive treatment less likely, as does the research cited earlier which has tended to report findings specific to C-ACEIs. C-ACEIs and acetylcholinesterase inhibitors may therefore have mutually promoting actions on acetylcholine bioavailability, and consequently cognitive function, but this requires further investigation.

There is evidence that certain C-ACEIs (captopril, trandolapril and ramipril) and NC-ACEIs (enalapril) are associated with reduced all-cause and cardiovascular mortality from 18\% to $40 \%$ among patients with hypertension. ${ }^{43}$ However, ACEIs have been found to be associated with an increased risk of mortality in patients with Alzheimer's disease compared with those receiving angiotensin II receptor blockers or other antihypertensives and also shown that initial protective effect of ACEIs was disappeared after adjusting for protopathic bias. ${ }^{29}$ In our study we found no differences in survival between groups, which might possibly be explained by a balancing of these risk and protective effects.

Regarding strengths of our study, we were able to assemble a large sample of patients with newly diagnosed Alzheimer's disease and homogeneity of the comparison groups, as well as outcome data availability, was maximised by restricting to people receiving acetylcholinesterase inhibitor treatment. We were also able to obtain large numbers of cognitive function recordings and to follow patients up for 3 years after dementia diagnosis: a substantially greater period than most previous studies. Limitations are principally related to the fact that the data-set was derived from routine records-derived information rather than a 'de novo' research cohort. For example, MMSE scores were a relatively crude measure of cognitive function, diagnoses were clinical rather than research quality, and confounding factors were limited by available data. In addition, the group receiving NC-ACEIs was relatively small and could not be analysed in detail. Finally, the classification of centrally and non-centrally active agents was based predominantly on animal models because human data have been generally lacking and heterogeneous. ${ }^{33}$ Although a compound's ability to cross the blood-brain barrier largely depends on its size, charge and lipophilicity, the integrity of the blood-brain barrier and the dose of the medication are also potentially influential. ${ }^{11}$ Importantly, all these limitations would be expected to obscure rather than exaggerate observed associations, and it is noteworthy that positive findings were still found to be present.

In summary, we found some evidence to support a timelimited effect of C-ACEIs on the trajectory of cognitive function in people with Alzheimer's disease receiving acetylcholinesterase inhibitor treatment. Visual inspection of the MMSE score trajectories in Fig. 1 suggests that cognitive function in people receiving C-ACEIs was either stable or improved compared with that at baseline for around 18 months after diagnosis, compared with around 9 months in those not receiving ACEIs. Whether this translates into delayed dependency and other functional outcomes requires further research, which is also required in order for C-ACEIs to be considered as treatment in the early stages of Alzheimer's dementia. ${ }^{44}$ As Rygiel has suggested, in clinical practice, practical suggestions for older people at risk of dementia that have hypertension or other medical conditions with indications for ACEIs, could be started on a C-ACEI instead of an NC-ACEI. ${ }^{44}$
Karim Fazal, MRCPsych, South West London and St George's Mental Health NHS Trust, London, UK; Gayan Perera, PhD, Institute of Psychiatry, Psychology and Neuroscience, King's College London, London, UK; South London and Maudsley NHS Foundation Trust, London, UK; Mizanur Khondoker, PhD, Norwich Medical School, University of East Anglia, Norwich Research Park, Norwich, UK; Robert Howard, MD, MRCPsych, Division of Psychiatry, University College London, London, UK; Robert Stewart, MD, FRCPsych, Institute of Psychiatry, Psychology and Neuroscience, King's College London, London, UK; South London and Maudsley NHS Foundation Trust, London, UK

Correspondence: Gayan Perera, Department of Psychological Medicine (Box 92), Institute of Psychiatry, Psychology and Neuroscience, King's College London, De Crespigny Park, London SE5 8AF, UK. Email: gayan.perera@kcl.ac.uk

First received 19 Oct 2016, final revision 7 Jun 2017, accepted 8 Jun 2017

\section{Funding}

The data resource, G.P. and R.S. are funded by the National Institute for Health Research (NIHR) Biomedical Research Centre at South London and Maudsley NHS Foundation Trust and King's College London. The views expressed are those of the authors and not necessarily those of the NHS, the NIHR or the Department of Health. G.P. additionally was funded by the British cross-research council Lifelong Health and Wellbeing (LHW) programme under Extending Working Lives as part of an interdisciplinary consortium on Wellbeing, Health, Retirement and the Lifecourse (WHERL) (ES/L002825/1). R.H. is supported by the UCLH NIHR Biomedical Research Centre.

\section{References}

1 Whitmer R, Sidney S, Selby J, Johnston SC, Yaffe K. Midlife cardiovascular risk factors and risk of dementia in late life. Neurology 2005; 64: 277-81.

2 Rozzini L, Chilovi BV, Bertoletti E, Conti M, Del Rio I, Trabucchi M, et al. Angiotensin converting enzyme (ACE) inhibitors modulate the rate of progression of amnestic mild cognitive impairment. Int J Geriatr Psychiatry 2006; 21: 550-5.

3 Hajjar I, Hart M, Chen YL, Mack W, Novak V, Chui CH, et al. Antihypertensive therapy and cerebral hemodynamics in executive mild cognitive impairment: results of a pilot randomized clinical trial. J Am Geriatr SOC 2013; 61: 194-201.

4 Gao Y, O'Caoimh R, Healy L, Kerins DM, Eustace J, Guyatt G, et al. Effects of centrally acting ACE inhibitors on the rate of cognitive decline in dementia. BMJ Open 2013; 3: e002881

5 Skoog I. The relationship between blood pressure and dementia: a review. Biomed Pharmacother 1997; 51: 367-75.

6 Hanon O, Seux ML, Lenoir H, Rigaud AS, Forette F. Hypertension and dementia. Curr Cardiol Rep 2003; 5: 435-40.

7 Launer L, Ross GW, Petrovitch H, Masaki K, Foley D, White LR, et al. Midlife blood pressure and dementia: The Honolulu-Asia aging study. Neurobiol Aging 2000; 21 $49-55$.

8 Poon IO. Effects of antihypertensive drug treatment on the risk of dementia and cognitive impairment. Pharmacotherapy 2008; 28: 366-75.

9 De Oliveira FF, Bertolucci PH, Chen ES, Smith MC. Brain-penetrating angiotensinconverting enzyme inhibitors and cognitive change in patients with dementia due to Alzheimer's disease. J Alzheimers Dis 2014; 42 (suppl 3): S321-4.

10 Ohrui T, Tomita N, Sato-Nakagawa T, Matsui T, Maruyama, M, Niwa K, et al. Effects of brain penetrating ACE inhibitors on Alzheimer disease progression. Neurology 2004; 63: 1324-5.

11 Sink KM, Leng X, Williamson J, Kritchevsky SB, Yaffe K, Kuller L, et al. Angiotensinconverting enzyme inhibitors and cognitive decline in older adults with hypertension: results from the Cardiovascular Health Study. J. Arch Intern Med 2009; 169 1195-202.

12 Hebert PL, McBean AM, O'Connor H, Frank B, Good C, Maciejewski ML. Time until incident dementia among Medicare beneficiaries using centrally acting or non-centrally acting ACE inhibitors. Pharmacoepidemiol Drug Saf 2013; 22: 641-8.

13 Ohrui T, Matsui T, Yamaya $M$, Arai $H$, Ebihara $S$, Maruyama $M$, et al. Angiotensinconverting enzyme inhibitors and incidence of Alzheimer's disease in Japan. J Am Geriatr SOC 2004; 52: 649-50.

14 Li NC, Lee A, Whitmer RA, Kivipelto M, Lawler E, Kazis LE, et al. Use of angiotensin receptor blockers and risk of dementia in a predominantly male population: prospective cohort analysis. BMJ 2010; 340: b5465.

15 Maxwell CJ, Hogan DB. Antihypertensive agents and prevention of dementia. BMJ 2010; 340: b5409.

16 Opie LH. Inhibition of the cerebral renin-angiotensin system to limit cognitive decline in elderly hypertensive persons. Cardiovasc Drugs Ther 2011; 25: 277-9.

17 Fernandes AC, Cloete D, Broadbent MTM, Hayes RD, Chang C-K, Roberts A, et al. Development and evaluation of a de-identification procedure for a case register 
sourced from mental health electronic records. BMC Med Inform Decis Mak 2013; 13: 71 .

18 Stewart R, Soremekun M, Perera G, Broadbent M, Callard F, Denis M, et al. The South London and Maudsley NHS Foundation Trust Biomedical Research Centre (SLAM BRC) Case Register: development and descriptive data. BMC Psychiatry 2009; 9: 51.

19 Hayes RD, Chang $C-K$, Fernandes A, Broadbent M, Lee $W$, Hotopf $M$, et al. Associations between substance use disorder sub-groups, life expectancy and all-cause mortality in a large British specialist mental healthcare service. Drug Alcohol Depend 2011; 118: 56-61.

20 Chang C-K, Hayes RD, Perera G, Broadbent MTM, Fernandes AC, Lee WE, et al Life expectancy at birth for people with serious mental illness from a secondary mental health care case register in London, UK. PLOS One 2010; 6: e19590.

21 Perera $G$, Khondoker $M$, Broadbent $M$, Breen $G$, Stewart R. Factors associated with response to acetylcholinesterase inhibition in dementia: a cohort study from a secondary mental health case register in London. PLOS One 2014; 9: e109484.

22 Sultana J, Chang C-K, Hayes RD, Broadbent M, Stewart R, Corbett A, et al. Associations between risk of mortality and atypical antipsychotic use in vascular dementia: a clinical cohort study. Int J Geriatr Psychiatry 2014; 29: 1249-54.

23 Ward G, Perera G, Stewart R. Predictors of mortality for people aged over 65 years receiving mental healthcare for delirium, in a south London Mental Health Trust, UK: a retrospective survival analysis. Int J Geriatr Psychiatry 2015; 30: 639-46.

24 Perera G, Broadbent M, Callard F, Chang C-K, Downs J, Dutta R, et al. Cohor profile of the South London and Maudsley NHS Foundation Trust Biomedical Research Centre (SLaM BRC) Case Register: current status and recent enhancement of an Electronic Mental Health Record derived data resource. BMJ Open 2016; 6: e008721.

25 Folstein MF, Folstein SE, McHugh PR. Mini-Mental State: a practical method for grading the cognitive state of patients for clinicians. J Psychiatr Res 1975; 12 189-98.

26 Rigby RA, Stasinopoulos DM. Generalized additive models for location, scale and shape. Appl Stat 2005; 54; 507-54.

27 Tzourio C, Anderson C, Chapman N, Woodward M, Neal B, MacMahon S, et al. Effects of blood pressure lowering with perindopril and indapamide therapy on dementia and cognitive decline in patients with cerebrovascular disease. Arch Intern Med 2003; 163: 1069-75.

28 O'Caoimh R, Healy L, Gao Y, Svendrovski A, Kerins DM, Eustace J, et al. Effects of centrally acting angiotensin converting enzyme inhibitors on functional decline in patients with Alzheimer's disease. J Alzheimers Dis 2014; 40: 595-603.

29 Kehoe PG, Davies NM, Martin RM, Ben-Shlomo Y. Angiotensin targeting antihypertensive drugs with mortality and hospitalization in primary care patients with dementia. J Alzheimers Dis 2013; 33: 999-1008.

30 Zou K, Liu J, Watanabe A, Hiraga S, Liu S, Tanabe C, et al. Abeta43 is the earliestdepositing Abeta species in APP transgenic mouse brain and is converted to Abeta41 by two active domains of ACE. Am J Pathol 2013; 182: 2322-31.
31 Ruitenberg A, den Heijer T, Bakker SL, van Swieten JC, Koudstaal PJ, Hofman A et al. Cerebral hypoperfusion and clinical onset of dementia: the Rotterdam Study. Ann Neurol 2005; 57: 789-94.

32 Savaskan E, Hock C, Olivieri G, Bruttel S, Rosenberg C, Hulette C, et al. Cortical alterations of angiotensin converting enzyme, angiotensin II and AT1 receptor in Alzheimer's dementia. Neurobiol Aging 2001; 22: 541-6.

33 Wright JW, Reichert JR, Davis CJ, Harding JW. Neural plasticity and the brain reninangiotensin system. Neurosci Biobehav Rev 2002; 26: 529-52.

34 Tuppo EE, Arias HR. The role of inflammation in Alzheimer's disease. Int J Biochem Cell Biol 2005; 37: 289-305.

35 Yaffe K, Lindquist K, Penninx BW, Simonsick EM, Pahor M, Kritchevsky S, et al. Inflammatory markers and cognition in well-functioning African-American and white elders. Neurology 2003; 61: 76-80.

36 Xue B, Zhang Z, Roncari CF, Guo F, Johnson AK. Aldosterone acting through the central nervous system sensitizes angiotensin II-induced hypertension. Hypertension 2012; 60: 1023-30

37 Wright JW, Kawas LH, Harding JW. A role for the brain RAS in Alzheimer's and Parkinson's diseases. Front Endocrinol (Lausanne) 2013; 4: 158

38 Dong $Y F$, Kataoka $K$, Tokutomi $Y$, Nako $H$, Nakamura $T$, Toyama $K$, et al. Perindopril, a centrally active angiotensin-converting enzyme inhibitor, prevents cognitive impairment in mouse models of Alzheimer's disease. FASEB J 2011; 25: 2911-20.

39 Tota S, Nath C, Najmi AK, Shukla R, Hanif K. Inhibition of central angiotensin converting enzyme ameliorates scopolamine induced memory impairment in mice: role of cholinergic neurotransmission, cerebral blood flow and brain energy metabolism. Behav Brain Res 2012; 232: 66-76.

40 Wyss JM, Kadish I, van Groen T. Age-related decline in spatial learning and memory: attenuation by captopril. Clin Exp Hypertens 2003; 25: 455-74.

41 Barnes JM, Barnes NM, Costall B, Horovitz ZP, Ironside JW, Naylor RJ, et al. Angiotensin II inhibits cortical cholinergic function: implications for cognition. J Cardiovasc Pharmacol 1990: 16: 234-8.

42 Yamada K, Horita T, Takayama M, Takahashi S, Takaba K, Nagata Y, et al. Effect of a centrally active angiotensin converting enzyme inhibitor, perindopril, on cognitive performance in chronic cerebral hypo-perfusion rats. Brain Res 2011; 1421: $110-20$

43 Ferrari R, Boersma E. The impact of ACE inhibition on all-cause and cardiovascular mortality in contemporary hypertension trials: a review. Expert Rev Cardiovasc Ther 2013; 11: 705-17.

44 Rygiel K. Can angiotensin-converting enzyme inhibitors impact cognitive decline in early stages of Alzheimer's disease? An overview of research evidence in the elderly patient population. J Postgrad Med 2016; 62: 242-8. 